\title{
EchoGéo
}

$7 \mid 2008$

Les nouveaux enjeux régionaux dans l'océan Indien occidental

\section{Le Great Limpopo Transfrontier Park}

Une gestion régionalisée de la biodiversité au service du développement?

\section{Nadia Belaidi}

\section{OpenEdition}

\section{Journals}

Édition électronique

URL : https://journals.openedition.org/echogeo/8523

DOI : $10.4000 /$ echogeo.8523

ISSN : 1963-1197

\section{Éditeur}

Pôle de recherche pour l'organisation et la diffusion de l'information géographique (CNRS UMR 8586)

Référence électronique

Nadia Belaidi, « Le Great Limpopo Transfrontier Park », EchoGéo [En ligne], 7 | 2008, mis en ligne le 16 octobre 2008, consulté le 31 juillet 2021. URL : http://journals.openedition.org/echogeo/8523 ; DOI : https://doi.org/10.4000/echogeo.8523

Ce document a été généré automatiquement le 31 juillet 2021.

EchoGéo est mis à disposition selon les termes de la licence Creative Commons Attribution - Pas d'Utilisation Commerciale - Pas de Modification 4.0 International (CC BY-NC-ND) 


\section{Le Great Limpopo Transfrontier Park}

Une gestion régionalisée de la biodiversité au service du développement?

\section{Nadia Belaidi}

1 Le Great Limpopo Transfrontière Park (GLTP) est un Parc pour la Paix qui réunit le Parc National Kruger en Afrique du Sud, le Parc National du Limpopo au Mozambique et le Parc National du Gonarezhou au Zimbabwe. L'accord trilatéral a été signé le 10 novembre 2000 et l'ouverture du Parc transfrontalier a commencé en 2004-2005. 
Illustration 1 - Carte de localisation

2

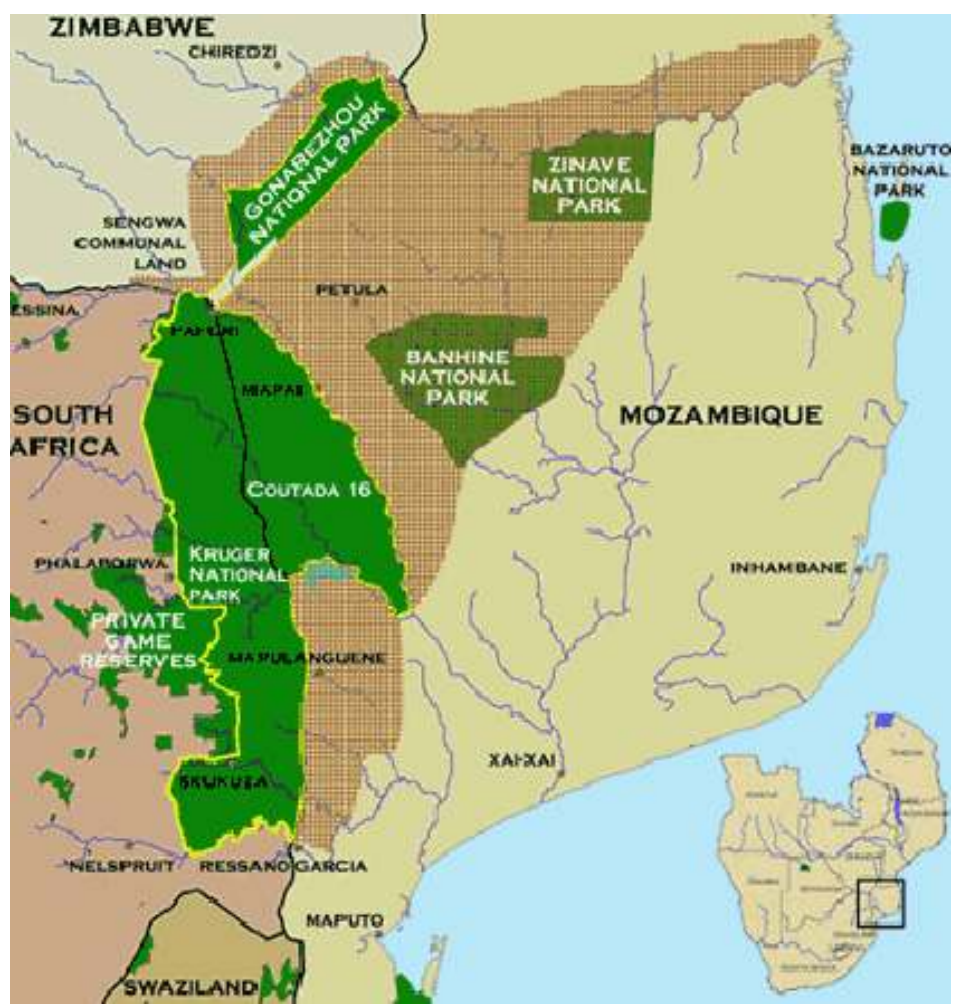

Source: Peace Park Foundation - Great Limpopo

Le GLTP est symbolique par :

- sa richesse biologique,

- sa superficie : environ $35000 \mathrm{~km}^{2}$ de parcs insérés dans une zone de conservation de près de $100000 \mathrm{~km}^{2}$ répartie sur trois pays,

- le fait que la zone frontalière des trois pays a été longtemps une zone de combats et d'infiltration militaire : le Mozambique et le Zimbabwe ayant tenu une place active dans la « ligne de front » contre l'apartheid. pour la Paix. Il est bien un « sous-ensemble particulier d'aires protégées où il y a:

- un objectif clair de protection de la biodiversité,

- une coopération entre au moins deux pays ou autorités sous-nationales,

- un objectif clair de paix $»^{1}$. diversité biologique et des ressources naturelles et culturelles qui y sont associées, ainsi qu'à la promotion de la coopération et de la paix.

6 La paix envisagée ne fait pas seulement référence au registre militaire en visant le contraire de la guerre. On vise, ici, la paix sociale, économique et culturelle. La logique de construction de ce Parc repose sur l'idée que l'articulation des zones de conservation facilite la résolution de conflits territoriaux - de toutes sortes - en transformant les préoccupations environnementales en objet de coopération entre des juridictions politiques diverses. Si les aires protégées apparaissent comme un outil important de protection et de maintien de la biodiversité dans les stratégies de conservation globales, leur but écologique n'est neutre ni socialement ni politiquement. Les 
différents rôles politiques des aires protégées relèvent d'un construit social, culturel et économique ${ }^{2}$. Or, dans beaucoup de pays en voie de développement, l'inégalité sociale, la pauvreté, la contestation des droits sur les ressources, la corruption, les tensions ethniques et le passé colonial peuvent créer des situations où les aires protégées agissent comme des mécanismes de contrôle et de pouvoir sur les ressources. Le concept de Parc pour la Paix est alors un véritable défi tant les différents rôles que les aires protégées peuvent revêtir en font des objets politiques qui peuvent mener à la résistance et au conflit. ${ }^{3}$

7 Pour atteindre l'objectif de Paix civile, sociale, économique et culturelle au travers de la "paix écologique", les Parcs pour la Paix s'appuient sur une coopération internationale qui possède des caractéristiques particulières :

- une interaction entre des juridictions multiples : gouvernements, société militaire, société civile, communauté scientifique, praticiens de la conservation,

- des juridictions qui agissent sur les zones de conservation nombreuses et de statuts divers que le parc réunit :

- Aires protégées de statut international : zones humides protégées par la Convention de

Ramsar, sites classés au Patrimoine Mondial de l'UNESCO, réserves de biosphères, habitat

d'espèces protégées par la convention CITES...

- Aires protégées de statut national : parc national, réserve, conservancy, safary aerea...

8 - des juridictions qui agissent sur des échelles spatiales (internationale, nationale, locale) différentes que les Parc pour la Paix articulent,

9 - des articulations qui ont pour fonction de négocier des dispositions efficaces pour protéger l'environnement.

10 C'est précisément le schéma adopté pour la constitution du GLTP. Or nous avons montré dans de récents travaux ${ }^{4}$ que ces articulations ne sont pas sans effets juridiques et politiques. Il en ressort un mécanisme de création de la règle qui semble particulièrement adapté pour régler les conflits entre les intérêts a priori contradictoires liés à la constitution d'aires protégées transfrontalières - cadre des Parcs pour la Paix. Ce mécanisme, qui vise une valorisation sociale de la protection de l'environnement, peut permettre d'atteindre la Paix recherchée et ainsi favoriser le processus de développement. Aussi peut-on se demander si le GLTP en constitue une illustration concrète ? Cette question nécessite d'abord d'expliciter le fondement juridique du mécanisme - l'ordre public écologique (I) - pour, dans un deuxième temps apprécier, par une analyse critique à l'aide de l'exemple du GLTP, comment l'objectif de Paix peut être atteint et favoriser le développement (II).

\section{I - L'ordre public écologique : une valorisation sociale de la protection de l'environnement par une articulation entre les échelles spatiales}

11 L'analyse de la recomposition spatiale effectuée dans le cadre du GLTP comme support à l'émergence d'un mécanisme de valorisation sociale de la protection de l'environnement dans cette région d'Afrique australe nécessite d'expliciter le concept juridique sur lequel ce mécanisme repose: l'ordre public écologique (A), ainsi que ses conditions d'émergence (B). 


\section{A - Le concept d'ordre public écologique}

12 L'effectivité du discours relatif au maintien de la paix civile, sociale, économique et culturelle par la protection de la biodiversité suppose que l'épuisement des ressources naturelles, par exemple, soit envisagé comme un risque tant du point de vue écologique que social, culturel ou économique. Or, dans sa conception lato sensu, le risque permet de relever que l'occurrence d'un événement aléatoire ne donnera lieu à contestation que s'il porte atteinte à des valeurs ou à des intérêts. La notion de risque ne devient effective que dès lors que la survenance possible du risque suspecté conduit à s'interroger sur l'opportunité de s'en protéger. ${ }^{5}$ Cela signifie que, pour que l'épuisement des ressources naturelles dans notre exemple mène à la coopération prévue dans les Parcs pour la Paix, la protection de l'environnement doit être considérée comme une valeur sociale essentielle ${ }^{6}$.

Le droit offre un «outil » particulièrement adapté pour désigner les valeurs les plus importantes pour la société : l'ordre public au sens matériel'7. L'ordre public est ici une "réponse juridique apportée à (...) un conflit de valeur ${ }^{8}$ : il indique ce qui relève des valeurs sociales essentielles d'une société donnée à une certaine période. De là, en repositionnant les valeurs et les concepts qui encadrent la prise de décisions et les relations politiques, l'ordre public matériel permet aussi de dépasser les seuls enjeux de pouvoirs et signale les valeurs sociales niées ou exclues. Ainsi, dans sa composante écologique, cet ordre public, qui permet de parler d'un impératif social de protection $\mathrm{du}$ milieu de vie contre les atteintes qu'il subit, peut souligner les contradictions qui traversent le système de protection de l'environnement et fournir les moyens de les surmonter.

14 Considéré au niveau international, le partage de « valeurs écologiques » a un impact sur la transcription et la mise en œuvre de ces mêmes valeurs : il peut faire apparaître le droit de l'environnement comme le protecteur d'un bien commun universel que les Etats ne pourraient ignorer et ainsi organiser un ordre juridique supérieur limitant la compétence de ces Etats. Il ne peut, bien évidemment, pas s'agir d'un droit inflexible tant le niveau local devient un élément nécessaire de mise en pratique des objectifs assignés au niveau international, même si le droit international reste essentiellement formé par la voie consensuelle du fait de l'égalité souveraine des Etats. Aussi, c'est précisément par l'articulation de ces deux niveaux que peut émerger « un ensemble de règles acceptées et reconnues par tous dont le but est de protéger les processus écologiques supports de toute vie dans la perspective d'assurer le développement durable et le bien-être de l'humanité »": un ordre public écologique. Nous avons, en effet, montré que le processus d'internationalisation des concepts du droit de l'environnement associé à la participation de la société civile fait naître un mécanisme de création de la règle susceptible de rendre la norme internationale de protection de l'environnement identifiable au plan local. Celle-ci peut ainsi être réappropriée et renforcée par ce niveau et devenir une pratique adaptée à ses spécificités sans pour autant être dénaturée.

\section{B - Les conditions d'émergence d'un ordre public écologique}

Deux conditions sont nécessaires pour voir émerger un ordre public écologique - une protection de l'environnement en tant que valeur sociale essentielle : internationalisation des concepts du droit de l'environnement (1) et une participation 
effective de la Société civile (2). C'est à dessein que les échelles d'intervention ne sont pas précisées, il s'agit en l'espèce de montrer l'importance d'articuler les deux niveaux.

\section{Internationalisation des concepts du droit de l'environnement} des instruments internationaux multiples mis en application par les gouvernements nationaux. Cette volonté des Etats de se donner des «lois» communes dans les conventions multilatérales découle de la prise de conscience de leur interdépendance. Malgré les faiblesses de l'ordre juridique international, la progression de cette interdépendance a permis l'apparition des notions de règles impératives (qui relèvent de l'ordre public matériel) et d'obligations erga omnes (obligations à l'égard de tous). Ces constructions juridiques dénotent une évolution philosophique de la Société internationale qui est en train de changer la nature du droit international. Le passage du national à l'international produit des conséquences juridiques - l'internationalisation $d u$ droit - qui permettent à la mise en place des normes mondiales de trouver toute sa signification : la recherche d'un état souhaitable qui n'existe pas encore. appartient aux entités souveraines - des questions les plus importantes qui faisaient auparavant partie du domaine réservé des Etats. L'internationalisation suppose l'existence d'un rapport dialectique entre les droits nationaux et le droit international. Ainsi, l'internationalisme n'est pas le rejet de l'idée de Nation, mais l'ouverture à l'humanité toute entière et à la solidarité entre les peuples..$^{10}$ De là, même si la vie internationale est dominée par les rapports interétatiques, un nouveau cadre institutionnel est progressivement apparu : la communauté internationale.

18 La société des Etats, telle que la connaissait le droit international classique, ne se réduit plus seulement à l'Etat et à sa souveraineté. Elle regroupe et dépasse aujourd'hui les sociétés dans leur cadre étatique dans la mesure où les Etats se sont imbriqués dans une communauté constituée des Etats et de leurs ressortissants: la Communauté internationale. Cette notion met l'accent sur ce qui rassemble plutôt que sur ce qui sépare. La Société internationale tend ainsi à être envisagée comme une collectivité humaine dont le caractère global oblige à une approche multilatérale du droit international. Cela signifie que la société internationale est aujourd'hui tenue par un lien de solidarité spécifique qui dérive des rapports internationaux, c'est-à-dire des échanges de tous genres entre individus appartenant à des sociétés politiques différentes. Ce lien de solidarité, en tant que fait, rejoint la norme juridique par l'intermédiaire de la "conscience sociale", qui permet à la solidarité de se transformer en règle de droit ${ }^{11}$ conduisant, ainsi, les Etats et les peuples à converger vers la promotion et la protection des valeurs sociales communes essentielles tout en conservant leur diversité. C'est, donc, en fonction des vues de la société actuelle que le système juridique est ordonné et impose le respect de cet « ordre juridique » à tous les acteurs de la vie sociale. C'est pourquoi la participation est un élément essentiel du système de valorisation sociale de la protection de l'environnement.

\section{Participation de la « Société civile »}

19 La participation permet de relever l'expression de la Société civile que nous entendons ici comme les groupes sociaux, mouvements, organisations et associations non 
étatiques et non économiques. Cette Société civile peut exprimer soit certaines valeurs soit une certaine manière d'envisager l'ordre social donnant ainsi naissance à une opinion publique (internationale) susceptible de donner corps à de nouvelles règles. Le droit enseigne que si cette expression prend un caractère collectif, constant et inscrit dans une certaine durée, elle peut constituer le vecteur de diffusion dans la société interétatique de représentations communes. Ainsi, envisager la Société internationale comme une collectivité humaine est une évolution significative : on passe de la Société internationale, celle que forme l'ensemble des Etats et des organisations internationales qu'ils créent, à la Communauté internationale qui marque l'existence d'intérêts communs et à laquelle participent, notamment, les populations. Cette distinction, entre Société internationale et Communauté internationale, ne relève pas de la simple nuance. Elle peut être le pivot du renforcement de la valeur sociale que représente un environnement de qualité. Cette évolution est, en effet, susceptible de donner naissance à de nouvelles formes de création juridique, de raviver la discussion sur l'organisation de la vie internationale et de fournir les moyens de répondre aux finalités de la protection de l'environnement.

Concrètement, le rapprochement opéré entre les « ordres juridiques » atteste déjà de la formation progressive d'une communauté de valeurs dont l'expression apparaît dans l'interdépendance nouvelle des Etats et l'incorporation de règles dans le droit interne par ce biais. Celui-ci permet de dépasser la construction conventionnelle strictement interétatique, pour accéder à un mode de production juridique encadré. Cependant, la participation des populations permet d'aller plus loin. Si l'internationalisation du droit diffuse les concepts du droit de l'environnement à travers les différentes échelles, la participation des populations - qu'elle soit organisée comme celle des associations ou non organisée comme celle de l'individu - peut prescrire la production juridique. Les personnes (physique ou morale) participent déjà de manière indirecte à la sanction du droit international, notamment par le biais des voies de droit qui leur sont ouvertes au sein de certaines juridictions internationales. Elles peuvent toutefois participer directement à son application et contribuer aussi à le (re)formuler. On estime, en effet, qu'elles participent à la création du droit dans la mesure où elles contribuent à l'élaboration d'une pratique constante qui participe à la cristallisation de normes coutumières. Leur intervention peut soit impulser de nouvelles règles soit fortifier celles qui existent et, ainsi, donner naissance à des normes de comportements adaptées aux problèmes environnementaux qui seront par la suite diffusées par le phénomène d'internationalisation du droit.

21 La recomposition territoriale qui donne naissance au GLTP met en scène tant l'internationalisation que la participation. Ce parc pour la Paix devient un bon outil d'analyse pour vérifier l'hypothèse contenue dans le mécanisme de l'ordre public écologique et apprécier si et comment l'objectif de Paix peut être atteint et favoriser le développement.

\section{II - La Paix pour atteindre le développement (durable) ? L'expérience de la recomposition territoriale du GLTP}

22 Le GLTP mesure environ $35000 \mathrm{~km}^{2}$. Il réunit plusieurs aires de conservation : le Parc national du Limpopo au Mozambique, le Parc national Kruger et le secteur Makuleke en 
Afrique du Sud, et le Parc national Gonarezhou, l'aire de Safari Malipati, le Sanctuaire Manjinji et le couloir Sengwe au Zimbabwe.

La constitution du GLTP s'est effectuée par la liaison entre, d'une part, le Parc national du Gonarezhou et le Parc national Kruger et, d'autre part, par l'unification du Parc national du Limpopo et du Parc national Kruger ${ }^{12}$. Nous nous intéresserons principalement à la liaison entre le Mozambique et l'Afrique du Sud.

Afin de constituer le GLTP, les autorités du Mozambique ont élevé une concession de chasse au statut de Parc national ${ }^{13}$ donnant ainsi naissance au Parc national du Limpopo le 27 novembre 2001. La jonction de ce «nouveau " parc avec le Parc national Kruger implique de recaser ${ }^{14}$ approximativement 6500 personnes. Selon le gouvernement mozambicain, les populations seront recasées en dehors du parc sur une base volontaire, probablement au sud du barrage de Massingir dans la zone de Gaza. Le cas du Mozambique interpelle notre problématique de l'émergence d'un système de protection de l'environnement en tant que valeur sociale essentielle sur ses deux conditions essentielles :

- le processus d'internationalisation qui est ici court-circuité par le phénomène de mondialisation économique (A).

- la participation des communautés locales qui, ici, sont évincées de la négociation (B).

\section{A - Un processus d'internationalisation court-circuité par le phénomène de mondialisation économique}

Depuis le milieu des années 1990, le gouvernement mozambicain a entrepris des changements législatifs et institutionnels importants afin d'appliquer les instruments internationaux de protection de l'environnement tel que la Convention sur la Diversité Biologique (CDB) que le Mozambique a ratifié en $1995^{15}$. C'est sur cette base que le Parc national du Limpopo a été incorporé au GLTP ${ }^{16}$. L'incorporation de cette zone dotée d'un potentiel important pour développer le tourisme, selon la Banque Mondiale,est présentée par les promoteurs du GLTP comme une occasion de combiner la conservation de la diversité biologique et le développement durable. Dans le cadre de la CDB, les éléments de la biodiversité sont perçus comme des éléments physiques originaux en interaction avec d'autres jouant un rôle particulier dans la dynamique des écosystèmes et des processus biologiques, au cœur d'interactions sur lesquelles reposent la vie (y compris humaine) et la matière ${ }^{17}$. Sur cette base, le choix de relier plusieurs zones de conservation ne devrait pouvoir être soumis à une mise en balance de la protection de l'environnement avec ce que ces zones représentent, à un moment donné, comme valeur monétaire, comme investissement fructueux à court terme.

Cependant, ici, le processus d'internationalisation des concepts du droit de l'environnement est court-circuité par le phénomène de mondialisation économique : l'ouverture à l'international s'effectue non pas en direction de la réglementation environnementale et de la protection des valeurs sociales qu'elle sous-tend mais vers la régulation par le niveau mondial ${ }^{18}$. Or les deux mécanismes ne se confondent pas. Là où le phénomène de mondialisation économique est soumis à des représentations issues du modèle économique dominant intériorisées et non remises en cause car elles paraissent être des évidences, des réalités dans une société donnée et à un moment donné ${ }^{19}$, le processus d'internationalisation du droit de l'environnement repose sur des approches théoriques, des modèles, des postulats liés à un système de valeur différent. 
La complexité de la réalité écologique a conduit à une réflexion épistémologique et une approche critique du modèle dominant qui a produit des concepts juridiques novateurs. La CDB est le résultat d'une évolution sur le plan juridique, elle-même reflétant une évolution de la perception des problèmes en cause et des solutions à leur apporter. Un des mérites de la convention est de fournir une vision globale regroupant tous les aspects de la diversité biologique. Ainsi, la CDB rappelle dès son préambule que les parties sont conscientes de "la valeur intrinsèque de la diversité biologique ». En tant qu'instrument juridique d'ensemble, elle sert de cadre à toute la réglementation internationale et en synthétise les principaux objectifs : le maintien des processus écologiques essentiels et des systèmes entretenant la vie; la préservation de la diversité génétique (dont dépend le fonctionnement de la plupart des processus et systèmes, la protection des plantes cultivées, des animaux domestiques et des microorganismes) ; l'utilisation durable des espèces et des écosystèmes dont sont tributaires les humains - aussi bien à la campagne que dans les zones industrielles.

La CDB n'est, toutefois, qu'une convention-cadre destinée à énoncer des principes. Chaque partie contractante choisit les moyens juridiques adaptés pour sa mise en application dans son ordre interne. Même si la convention porte les concepts et acquis juridiques en la matière et qu'elle peut, ainsi, être utilisée pour mettre en œuvre les évolutions en matière de biodiversité, elle reste soumise au droit souverain des Etats d'exploiter leurs propres ressources «selon leur politique d'environnement» (art. 3). La protection et l'utilisation de la biodiversité découlent de leur compétence sur toute l'étendue de leur territoire. Le processus d'internationalisation du droit est donc soumis à la volonté des Etats et à la hiérarchisation des valeurs sociales qu'ils effectuent. Dans notre cas d'étude, cette hiérarchisation a conduit à faire primer le potentiel touristique de la zone retenue sur le maintien de populations sur leur territoire. Mieux, alors que la composition du GLTP s'effectue au nom du caractère fédérateur prêté à la protection de la biodiversité pour lutter contre les conflits de toutes sortes et ainsi promouvoir le développement, les populations vivant dans ou autour des secteurs désignés pour constituer le «Parc pour la Paix» sont délocalisées sans prendre part à aucune négociation.

\section{B - Des communautés locales évincées de la négociation}

28 Les communautés locales faisant partie intégrante des projets de Parcs pour la Paix, on pouvait s'attendre à ce qu'elles interagissent avec les ONG locales et mondiales, les bailleurs et institutions financières internationales comme la Banque mondiale. Cependant, ces liens entre les communautés, les gouvernements, les organisations internationales et le secteur privé sont ici ambigus. De prime abord, il semblerait que la Banque mondiale ait subventionné le projet sans avoir la pleine connaissance de l'impact du GLTP sur les populations locales. Il est, cependant, peu vraisemblable que l'on ait découvert rétrospectivement que la zone était habitée : toutes les projections géographiques du GLTP incorporaient des secteurs occupés par des populations. On peut, d'ailleurs, supposer que la Banque mondiale était consciente que la zone dont le statut a été élevé au rang de Parc national était habitée puisqu'elle a alloué \$5 millions au programme pilote au Mozambique afin de contribuer à la réduction de la pauvreté et aider les communautés locales dans et autour des secteurs de conservation. Il est probable que les promoteurs du GLTP soient restés silencieux au sujet du devenir des personnes vivant à l'intérieur du GLTP afin d'affaiblir les résistances des communautés 
locales et des groupes de pression internationaux au moment où la création du GLTP s'avérait être une entreprise difficile au niveau intergouvernemental.

Bien que le premier directeur du Parc national du Limpopo affirme que la plupart des villageois est d'accord avec les plans du parc, il s'avère que les populations n'ont pas été réellement informées ou intégrées lors du processus de création du GLTP. Ainsi, malgré les déclarations publiques qui le présentent comme une initiative en faveur des communautés locales, les populations ont été laissées en dehors de son processus de création. Il n'y a, notamment, pas eu de consultation des communautés locales affectées par la création du Parc national du Limpopo. Il semblerait que les chefs traditionnels et élus aient été consultés concernant le lieu de recasement mais il demeure un obstacle. Certains chefs élus posent la condition du reclassement des populations sur une même zone afin qu'ils puissent conserver leur contrôle sur les communautés pour s'aligner sur les volontés du gouvernement. Ceci n'a, cependant, pas empêché le gouvernement mozambicain d'approuver une option de reclassement en 2004 à laquelle la banque de développement allemande (KfW) contribuera à hauteur de 6 millions d'euros. Cette contribution est présentée comme une aide au développement en faveur des communautés locales mais elle assure surtout l'ouverture du GLTP. De même, des ONG ont ouvert une lodge en mai 2004 dont les communautés locales doivent tirer bénéfice par l'écotourisme. ${ }^{20}$

Reprenant l'argument de la Banque Mondiale, l'écotourisme est présenté comme un vecteur de développement durable alors même que, pour les opérateurs économiques, l'association écodéveloppement-développement durable est en tout premier lieu une invitation à la promotion de la croissance économique. Même si on ne peut déduire de définition unique du développement durable du rapport Brundtland, on peut être certain que son caractère transversal - assis sur les trois facettes économique, sociale et écologique - vise au minimum l'intégration sociale des communautés locales et exclut toute forme d'exploitation ou de domination. Aussi, écarter les Communautés locales d'une négociation menée au nom du développement durable confirme la place donnée aux valeurs véhiculées par la mondialisation économique et montre comment les concepts $d u$ droit international de l'environnement peuvent être instrumentalisés pour cautionner un choix politique en contradiction avec leurs objectifs fondamentaux.

L'articulation des zones de conservation opérée dans le cadre de la construction du GLTP est présentée comme un moyen de faciliter la résolution de conflits territoriaux en favorisant le dialogue sur des intérêts communs. Toutefois, ces conflits sont inscrits dans les structurations sociales et culturelles, aussi le changement de forme et d'organisation doit aussi emporter un changement de contenu et de substance. Une recomposition territoriale qui a pour objet de transformer les préoccupations environnementales en objet de coopération entre diverses juridictions politiques doit envisager l'environnement pour tout ce qu'il est et pas seulement pour ce que l'on veut qu'il représente. Celui-ci ne se résume pas à être un support pour le tourisme, les "services environnementaux vitaux" sous-tendent - aussi - la garantie des droits humains ${ }^{21}$. Or les droits de la personne représentent les valeurs fondatrices de tout système juridique. C'est sur cette base que l'analyse du GLTP, comme support à l'émergence d'un ordre public écologique dans cette région d'Afrique australe, montre que sa construction, en favorisant les valeurs véhiculées par le phénomène de mondialisation notamment économique, néglige la garantie et le respect des " autres » valeurs sociales. Cette posture présente l'ouverture des frontières, opérée par le GLTP, 
non pas comme un vecteur de développement commun mais comme un enjeu de domination politique et économique.

Un détour par l'Afrique du Sud, avec laquelle s'effectue la constitution du GLTP par la liaison du Parc national Kruger avec les deux autres parcs, précise ces points. Le Parc pour la Paix du Great Limpopo traduit l'ouverture du territoire sud-africain à l'égard des pays voisins en s'appuyant sur une transformation de ses frontières. La nouvelle organisation régionale $\mathrm{du}$ territoire sud-africain, assise sur une approche supranationale, permet à l'Afrique du Sud de s'ériger en véritable puissance internationale, non seulement en Afrique australe mais aussi au niveau du développement africain dont elle tend à être considérée comme un moteur. Cela favorise, au plan mondial, son identification comme une des principales voix du Sud. A cela s'ajoute que la protection de la biodiversité, devenue slogan de "marketing vert ", soutient un processus de privatisation dans les parcs nationaux. Ainsi, dans le parc national Kruger, par exemple, si la gestion de la biodiversité continue à relever du domaine public (SANpark), la gestion financière du parc est abandonnée aux milieux d'affaire internationaux avec le support technique de la Banque Mondiale. Dans cette configuration, la signature d'accords qui promeuvent des TFCA (Transfrontier Conservation Area qui vise les Peace parks) exacerbe d'une part la concurrence entre les intérêts nationaux et régionaux en présence et, d'autre part, la priorité donnée à la valeur économique de la biodiversité au détriment des autres valeurs sociales qu'elle sous-tend, et cela en contradiction complète avec les objectifs affichés de ces recompositions territoriales.

\section{BIBLIOGRAPHIE}

Boutelet M. et Fritz J.-C. (dir.), 2005. L'ordre public écologique. Bruylant, Bruxelles

Belaidi N., 2008. La lutte contre les atteintes globales à l'environnement : vers un ordre public écologique? Bruylant, Bruxelles, $498 \mathrm{p}$.

Besançon Ch., Hammill A., 2007. "Measuring Peace Performance: Definitions and Experiences" in : Saleem A.H. (ed.), Peace Parks. Conservation and Conflict Resolution, The MIT Press, Cambridge, Massachusetts (USA) and London (U.K.), p. 23-40.

Commission Mondiale des Aires Protégées (CMAP), Sandwith Tr., Shine C., Hamilton L. and Sheppard D., 2001. Aires protégées transfrontalières pour la paix et la coopération. Collection Guides des meilleures pratiques pour les aires protégées nำ 7, IUCN - Union mondiale pour la nature, Gland.

Duffy R., 2007. "Peace parks and global politics: the paradoxes and challenges of global Governance", in : Saleem A.H. (ed.), Peace Parks. Conservation and Conflict Resolution, The MIT Press, Cambridge, Massachusetts (USA) and London (U.K.), p. 55-68.

Fritz J.-C., 2006. "Restructuration territoriale et identités sociales dans la nouvelle Afrique du Sud". Recherches internationales, $\mathrm{n}^{\circ}$ 78, 4-2006, p 73-114. 
Kiss A. et Beurier J.-P., 2000. Droit international de l'environnement, Pédone, Paris.

Ramutsindela M., 2007. Transfrontier Conservation in Africa. At the Confluence of Capital, Politics and Nature. CABI, Oxford.

Romano S., 1975. L'ordre juridique. Dalloz, Paris,.

Saleem A. H., 2007. "Introduction : A Natural Connection between Ecology and Peace ?", in : Saleem A.H. (ed.), Peace Parks. Conservation and Conflict Resolution, The MIT Press, Cambridge, Massachusetts (USA) and London (U.K.), , p. 1-18.

Saleem A.H. (ed.), 2007. Peace Parks. Conservation and Conflict Resolution. The MIT Press, Cambridge, Massachusetts (USA) and London (U.K.).

Peace Parks Foundation Annual Review, 1997-2006.

\section{NOTES}

1. Commission Mondiale des Aires Protégées (CMAP), Sandwith TR., Shine C., Hamilton L. and Sheppard D., Aires protégées transfrontalières pour la paix et la coopération, Collection Guides des meilleures pratiques pour les aires protégéesno 7, IUCN - Union mondiale pour la nature, Gland, 2001

2. Les Aires Protégées représentent différents « intérêts " pour des groupes différents : pour des défenseurs de l'environnement, elles constituent une mesure efficace pour protéger la biodiversité et la production de services environnementaux vitaux ; pour les Tour-opérateurs, c'est une base pour le développement de l'écotourisme; pour les sociétés pharmaceutiques, c'est une source d'informations génétiques pour le développement de médicaments; pour les sociétés minières et pétrolières, c'est une provision inexplorée de revenu; pour l'armée, c'est un refuge et une cible stratégique pendant les périodes de conflits ouverts; pour les communautés locales avoisinantes, les aires protégées peuvent limiter l'accès aux ressources de subsistance, entraîner des délocalisations ou générer des revenus par le tourisme.

3. V. BesançonCh.,LanjouwA.,HammillA., "Measuring Peace Performance: Definitions and Experiences", in Saleem A.H. (ed.), Peace Parks. Conservation and Conflict Resolution, The MIT Press, Cambridge, Massachusetts (USA) and London (U.K.), Prepublication Draft, August 17, 2005, p 28-29.

4. BELAIDI N., La lutte contre les atteintes globales à l'environnement : vers un ordre public écologique?, Bruylant, Bruxelles, 2008, 498 p.

On peut trouver le terme " ordre public écologique » dans la littérature française mais il n'a été défini que dans le cadre du droit interne français soit de manière explicite, Caballero F., Essai sur la notion juridique de nuisance, LGDJ, Paris, 1981, p. 19-176; Prieur M., Droit de l'environnement, Dalloz, $5^{\mathrm{e}}$ édition, Paris, 2004, p. 54-55, n 60-61; soit de manière implicite, Picard M., La notion de police administrative, Thèse Paris II, 1978, p. 226 ; expression proche, Ordre public de la nature, Prieur M., La pollution atmosphérique, Coll. Droit et économie de l'environnement, PPS, 1975, p. 5. Plus récemment et en liaison avec la vision renouvelée que nous proposons, Kiss A., "L'ordre public écologique : définition, L'ordre public écologique, Actes du Colloque organisé par le GIDE, les 6 et 7 février 2003 à Dijon, Bruylant, Bruxelles, 2005.

5. Boisson de Chazournes L., «Le principe de précaution : nature, contenu et limites » in Leben C. et Verhoeven J., Le principe de précaution. Aspects de droit international et communautaire, Actes du colloque organisé par l'Institut des hautes études internationales en coopération avec le Centre de droit européen de l'Université Panthéon-Assas (Paris II), le 5 mars 2001, Editions Panthéon-Assas, Paris, 2002., p. 71-75. 
6. V. Hofrichter R. (éd.), Reclaiming the Environmental Debate. The Politics of Health in a Toxic Culture, The MIT Press, Masschussets-London, 2002.

7. Il est à différencier de l'ordre public fonctionnel que l'on rencontre principalement en droit français et qui vise notamment le pouvoir et les règles de police qui protégent notamment la tranquillité, la salubrité et la sécurité.

8. Vincent-Legoux M.-C., L'ordre public. Etude de droit comparé interne, PUF, Paris, 2001, p. 526.

9. Belaidi N., La lutte contre les atteintes globales à l'environnement : vers un ordre public écologique?, Bruylant, Bruxelles, 2008, p. 461.

On peut trouver le terme "ordre public écologique » dans la littérature française mais il n'a été défini que dans le cadre du droit interne français (donc plutôt dans le logique fonctionnelle du terme) soit de manière explicite, Caballero F., Essai sur la notion juridique de nuisance, LGDJ, Paris, 1981, p. 19-176 ; Prieur M., Droit de l'environnement, Dalloz, 5 édition, Paris, 2004, p. 54-55, n 60-61 ; soit de manière implicite, Picard D M., La notion de police administrative, Thèse Paris II, 1978, p. 226 ; expression proche, Ordre public de la nature, Prieur M., La pollution atmosphérique, Coll. Droit et économie de l'environnement, PPS, 1975. 5. Plus récemment et en liaison avec la vision renouvelée que nous proposons, Kiss A., " "L’ordre public écologique : définition », L'ordre public écologique, Actes du Colloque organisé par le GIDE, les 6 et 7 février 2003 à Dijon, Bruylant, Bruxelles, 2005.

10. De là, une universalisation du droit international peut s'accomplir, c'est le cas avec l'adoption de la Charte des Nations-Unies (et le processus de décolonisation). Son produit final, c'est l'universalité voire la pluriversalité : l'existence de valeurs communes à tous les Etats et à tous les peuples. Il s'agit alors de la transformation des «biens» entre les mains de quelques-uns en «biens» accessibles à tous.

11. V. Romano S., L'ordre juridique, Dalloz, Paris, 1975.

12. Sur les modalités de liaisons voir Ramutsindela M., Transfrontier Conservation in Africa. At the Confluence of Capital, Politics and Nature, CABI, Oxford, 2007, p. 106-115.

13. Aire située à l'est du Parc national Kruger au Mozambique (Coutada 16).

14. On pourrait même dire « reclasser » tant le changement de territoire induit de changements sociaux et culturels.

15. Convention sur la diversité biologique, 5 juin 1992, Rio de Janeiro in BOISSON DE CHAZOURNES L., DESGAGNE R et ROMANO C, Protection internationale de l'environnement, Recueil d'instruments juridiques, Pédone, Paris, 1998, p. 230 sqq.; International Environnemental LawEnvironmental Multilateral Treaties n992, p. 42 sqq.

16. V. Republic of Mozambique, Ministry for Coordination of Environmental Affairs, Third National Report of the Convention on Biological Diversity, Maputo, 2006.

17. La CDB définit la diversité biologique comme étant « la variabilité des organismes vivants de toute origine y compris, entre autres, les écosystèmes terrestres, marins et autres écosystèmes aquatiques et les complexes écologiques dont ils font partie; cela comprend la diversité au sein des espèces et entre les espèces ainsi que celles des écosystèmes " (art. 2).

18. Voir sur ce point, DuffyR., "Peace parks and global politics: the paradoxes and challenges of global Governance", in Saleem A.H. (ed.), Peace Parks. Conservation and Conflict Resolution, The MIT Press, Cambridge, Massachusetts (USA) and London (U.K.), Prepublication Draft, August 17, 2005, p. 66-68.

19. Voir sur ce point Fritz G., "Science universelle et cultures des peuples » in Apostolidis C., Fritz G. et Fritz J.-C., L'humanité face à la mondialisation. Droits des peuples et environnement, L'Harmattan, Paris, 1997, p. 113-130 ; Fritz J.-C., « Mondialisation et destruction des cadres et des conditions de vie ", Santé publique, 2002, vol. 14, n 4, p. 425-464.

20. Sur les conséquences socio-spatiales des liaisons voir Ramutsindela M., Transfrontier Conservation in Africa. At the Confluence of Capital, Politics and Nature, op. cit. 
21. «les conditions et les processus à travers lesquels les écosystèmes naturels, et les espèces qui en font partie, soutiennent et alimentent la vie humaine ", Daily (G.C.), Nature's Services : Societal Dependence on Natural Ecosystems, Island Press, Washington D.C., 1997 V. aussi Study of Critical Environmental Problems (SCEP), Man's Impact on the Environment, MIT Press CambridgeMA,1970 ; Holdren (J.) \& Ehrlich (P.), "Human Population and the Global Environment", American Scientist, 1974-62, p. 282-292.

Ces services maintiennent la diversité biologique et la production de biens par les écosystèmes. Ils assurent des fonctions de support de vie: le maintien de l'équilibre naturel de l'environnement permet aux espèces animales et végétales de continuer à assurer leur fonction au sein des écosystèmes. Dans ce cadre, nier les rapports d'interactions entre les éléments de l'environnement et omettre qu'ils sont complémentaires, et que la protection de l'un entraîne nécessairement la protection des autres, aboutit à ne protéger que de manière très relative les droits de la personne.

\section{RÉSUMÉS}

Cet article a pour objet de présenter quelques problématiques d'une recherche sur la construction d'un mécanisme de valorisation sociale de la protection de l'environnement : l'ordre public écologique. Cette étude s'appuie sur une expérience récente et originale en Afrique australe : la création de Parcs pour la Paix dont relève le Great Limpopo Transfrontier Park. Ces parcs sont officiellement consacrés à la protection et à la conservation de la diversité biologique, des ressources naturelles et culturelles qui y sont associées, ainsi qu'à la promotion de la coopération et de la Paix qu'elle soit civile, sociale, économique ou culturelle. La logique de construction de ces parcs repose sur l'idée que l'articulation des zones de conservation facilite la résolution de conflits territoriaux - de toutes sortes - en transformant les préoccupations environnementales en objet de coopération entre des juridictions politiques diverses. C'est précisément le schéma du concept juridique objet de nos travaux : l'ordre public écologique. Aussi peut-on se demander si le GLTP, en particulier, (peut) constitue(r) une illustration concrète de ce mécanisme et permettre d'atteindre la Paix recherchée, favorisant ainsi les processus de développement?

This article aims to present some problems of researches on the construction of a mechanism of social valorisation of the environmental protection: ecological public order. This study leans on a recent and original experience in southern Africa: the creation of Peace Park like the Great Limpopo Transfrontier Park (GLTP). These parks are officially dedicated to the protection and to the preservation of biological diversity, natural and cultural resources which are associated with it, as well as in the promotion of the cooperation and the peace. The peace looked for in these parks is not only the opposite of the war but it is also social, economic and cultural peace. The logic of construction of these parks is based on the idea that the articulation of conservation areas facilitates the resolution of territorial conflicts - of all kinds - by transforming the environmental concerns into object of cooperation between several political jurisdictions. It is the plan of the ecological public order concept. Thus, we wonder if the GLTP, in particular, (can) establish a concrete illustration of this mechanism and if it (can) lead to Peace, facilitating in this way any process of development. 
INDEX

Mots-clés : articulation, biodiversité, développement, échelle spatiale, valeur sociale

Keywords : articulation, biological diversity, development, social value, spatial scale

\section{AUTEUR}

\section{NADIA BELAIDI}

Nadia Belaidi (nadia.belaidi@univ-paris1.fr) est chercheur CNRS, spécialiste en Droit international de l'environnement et est membre de l'UMR PRODIG 8586. 